\title{
EDITORIAL
}

\section{Forthcoming Changes to the Journal de Physique and the Revue de Physique Appliquée}

The work environment of European scientists is undergoing rapid transformation. Cooperation between scientists from diverse countries of the Community is becoming more frequent - either spontaneous or encouraged by the awarding of contracts for group research - and multinational outfits are taking up a growing importance in European research. And finally, in 1993, Economical and Political Europe will undergo an additional step toward unification. The considerable changes that have recently occured in Central Europe will also provide opportunities for new partnerships in the future Scientific Europe. In such a context, the maintenance of an editorial policy for national scientific publicications will have no effect in offsetting the importance of publications from large industrialized countries like the United States or Japan. Nor do we believe that any single country belonging to the Community is able to claim to have means of spreading other partners' results through its own publications. The only solution that seems reasonable for bringing together a large part of European production without engendering too much frustration is a Publishing Europe. This enterprise has already begun, by the way, with the publication of articles in the journal Europhysics Letters, an obvious success with European physicists and those of the rest of the world.

The French Physical Society and the CNRS supervise, through the medium of the Commission of French Publications of Physics, the two general journals, the Journal de Physique and the Revue de Physique Appliquée. These two journals are indeed of European vocation, for they are recognized by the European Physical Society. At the same time, European recognition of numerous journals, although signifying an evolution toward European unity, also indicates that we have not yet achieved this goal of unification.

To attempt to make French publications of physics important partners in Publishing Europe, the Commission of Publications has proposed a series of reforms that will take effect January 1, 1991. These reforms concern both the journals' structure and editorial policy. The desired effect is the strengthening of our publications in the areas that have traditionally constituted their commerce by increasing the number of articles and their distribution.

Before beginning an account of the measures taken, I would like to make a comment regarding the distribution of the Journal de Physique. Presently, there is a greater number of subscribers in the U.S. than in France. Distribution in France represents about $20 \%$ of the total, a figure approaching that of Europhysics Letters. We thus have an indication that the Jourhal is well received by the scientific community, as I hardly believe that North American librarians are philanthropists who subscribe their institutions to journals that will have no readers. Our goal is to double the current distribution.

Among the decisions made, first there is the one that will have the Journal appear in four sections: two sections for basic physics, one for applied physics and another for reviews of international proceedings.

Section I will regroup General Physics, Condensed Matter and interdisciplinary fields. Atomic and Molecular Physics, that of Agregates, Chemical Physics, Mechanics and Hydrodynamics will make up Section II. This division is based on the current weight of diverse headings. Of course, future modifications of these divisions in terms of the evolution of the subjects will not be excluded. We hope, despite this sectioning, that readers will continue to subscribe to the two sections of basic physics despite the fact that subscriptions will be available to each journal individually. Section III will publish in particular articles in the fields of Applied Physics, Material Sciences, 
Plasma Physics and Scientific Instrumentation as single theme issues. Section III of the Journal de Physique will replace the Revue de Physique Appliquée. The publication will be monthly each for sections I, II and III. The proceedings will appear in Section IV at the rate of 8 to 12 issues per year.

Short Communications will be published in Sections I and II (and eventually in Section III). Since rapid publication is the important advantage of the Short Communications (a maximum turnaround time of two months, from scientific acceptance to publication has been guaranteed by the Editions de Physique), proofs will not be sent to authors, and the communication will be published in the first issue to appear, independent of its speciality. Finally, a summary in French of articles written in English will no longer be required, a task often painful for some non French-speaking authors and for the editors of the Journal.

It must be pointed out that publishing in the Journal will remain free of charge (as is the case with all publications recognized by the European Physical Society), and 50 reprints will be sent to authors at no cost.

The work of scientific publishing will continue to be carried out by the group of scientific co-editors working in close collaboration with the secretariat of publications located at the University of Paris-Sud. However, since the goal of these reforms is to make the Journal more well known abroad and to facilitate the collection of a greater number of quality manuscripts, we have decided to adjoin international scientific editors to the group of French co-editors. The work of these editors will consist of evaluating manuscripts directly sent to them with the help of judges following the Journal's standard publishing procedure. The international editors will only forward accepted manuscripts to the Editions de Physique (via the secretariat of publications). We have already obtained accords of cooperation by well known scientists in the FRG, Italy, the United Kingdom, the USSR and Canada.

The Journal de Physique will remain a tool for the publication of leaders of a particularly open scientific spectrum, even if certain fields like Nuclear Physics and Plasma Physics are more often published in specialized journals.

Let us hope that these reforms will contribute to the greater recognition and appreciation of the Journal de Physique (the new model) to our foreign colleagues, and especially to our French colleagues (to which they submit hardly more than $5 \%$ of their scientific output). This set goal is not out of reach. Doubling the volume of publications will already be an initial satisfactory result. If this effort is not undertaken, there is the possibility of the development of scientific publishing monopolies that will more than likely have an adverse effect on the free distribution of information and even on scientific progress itself.

Denis Jérome

President

Publications Committee 